\title{
Catalytic, Asymmetric Preparation of Ketene Dimers from Acid Chlorides
}

\author{
Michael A. Calter* and Robert K. Orr \\ Department of Chemistry, University of Rochester, \\ Rochester, New York 14627-0216
}

Supporting Information

General Experimental Procedure: For general experimental procedures, see previously cited work. ${ }^{1}$

General procedure for In Situ Ketene Formation and Dimerization: To TMSQN

$(0.25 \mathrm{mmol})$ in anhydrous $\mathrm{CH}_{2} \mathrm{Cl}_{2}$ (solvent volume) under argon was added Hünig's base (5.0 $\mathrm{mmol})$, followed by the appropriate acid chloride $(5.0 \mathrm{mmol})$. After stirring for Time A at room temperature, $\mathrm{HN}(\mathrm{OMe}) \mathrm{Me}(0.18 \mathrm{~mL}, 0.15 \mathrm{~g}, 2.5 \mathrm{mmol})$ and hydroxypyridine $(0.024 \mathrm{~g}, 0.25 \mathrm{mmol})$ were added to the reaction mixture. The mixture was then stirred for Time $\mathrm{B}$ at room temperature, after which $\mathrm{pH} 7$ buffer solution concentrate $(10 \mathrm{~mL})$ was added. The organic layer was removed and the aqueous layer was extracted with $\mathrm{CH}_{2} \mathrm{Cl}_{2}(4 \times 50 \mathrm{~mL})$. The combined organic layers were dried $\left(\mathrm{Na}_{2} \mathrm{SO}_{4}\right)$, concentrated in vacuo, and the residue purified by flash chromatography on silica gel (EtOAc/hexanes).

General Procedure for Filtering Dimer Prior to Opening: Following Time A, the mixture was diluted with pentane $(50 \mathrm{~mL})$ and filtered through silica gel $(2 \mathrm{~g}$ in a $2 \mathrm{~cm}$ flash chromatography column) at a flow rate of $100 \mathrm{~mL} / \mathrm{min}$. The silica gel was then washed with a $1: 1 \mathrm{CH}_{2} \mathrm{Cl}_{2}$ :pentane mixture $(100 \mathrm{~mL})$ or until the yellow band reached the bottom of the column. To the resulting filtrate was added $\mathrm{HN}(\mathrm{OMe}) \mathrm{Me}(0.18 \mathrm{~mL}, 0.15$ $\mathrm{g}, 2.5 \mathrm{mmol})$ and hydroxypyridine $(0.024 \mathrm{~g}, 0.25 \mathrm{mmol})$. The mixture was stirred for Time B, after which $\mathrm{pH} 7$ buffer solution concentrate $(10 \mathrm{~mL})$ was added. The organic layer was removed and the aqueous layer was extracted with $\mathrm{CH}_{2} \mathrm{Cl}_{2}(4 \times 50 \mathrm{~mL})$. The combined organic layers were dried $\left(\mathrm{Na}_{2} \mathrm{SO}_{4}\right)$, concentrated in vacuo, and the residue purified by flash chromatography on silica gel (EtOAc/hexanes).

(S)-2-Methyl-3-oxo-pentanoic acid methoxy-methyl-amide, 2: Acid chloride = propionyl chloride, solvent volume $=50 \mathrm{~mL}$, Time $\mathrm{A}=6 \mathrm{~h}$, Time $\mathrm{B}=2 \mathrm{~h}$, yield $=0.137$ g, $79 \%$. This compound was previously characterized. ${ }^{2}$ HPLC analysis (Daicel Chiralpak OD-H, 98:2 hexanes: $i$-propanol, $0.5 \mathrm{~mL} / \mathrm{min}, 254 \mathrm{~nm}$ ) showed a 97:3 mixture of enantiomers $\left(\mathrm{R}_{\mathrm{t}(R)}=23.18 \mathrm{~min}, \mathrm{R}_{\mathrm{t}(S)}=25.41 \mathrm{~min}\right)$.

\footnotetext{
${ }^{1}$ Calter, M. A.; Liao, W.; Struss, J. A. J. Org. Chem. 2001, 66, 7500-7504.

${ }^{2}$ Calter, M. A.; Guo, X. J. Org. Chem. 1998, 63, 5308-5309.
} 
(S)-2-Ethyl-3-oxo-hexanoic acid methoxy-methyl-amide (4a). Acid chloride = butyryl chloride, solvent volume $=50 \mathrm{~mL}$, Time $\mathrm{A}=6 \mathrm{~h}$, Time $\mathrm{B}=2 \mathrm{~h}$, yield $=0.3423 \mathrm{~g}, 68 \%$. HPLC analysis (Daicel Chiralpak OD-H, 99:1 hexanes: $i$-propanol, 0.5 mL/min, $254 \mathrm{~nm}$ ) of the purified compound showed a 96:4 mixture of enantiomers $\left(\mathrm{R}_{\mathrm{t}(S)}=15.11 \mathrm{~min} ; \mathrm{R}_{\mathrm{t}(R)}\right.$ $=16.45):[\alpha]_{\mathrm{D}}{ }^{23}=-13.7^{\circ}\left(c 1.00, \mathrm{CHCl}_{3}\right)$; IR (neat film) $2964.2,1716.7,1663.3 \mathrm{~cm}^{-1} ;{ }^{1} \mathrm{H}$ NMR $\left(\mathrm{CDCl}_{3}, 400 \mathrm{MHz}\right) \delta 3.69(3 \mathrm{H}, \mathrm{s}), 3.63$ (app t, J = 3.2 Hz, 1H), 3.22 (s, 3H), 2.48$2.43(\mathrm{~m}, 2 \mathrm{H}), 1.95$ (app dp, J = 7.2, $14.6 \mathrm{~Hz}, 1 \mathrm{H}), 1.83$ (app dp, J = 7.0, 14.1 Hz, 1H), 1.61 (app sextet, $\mathrm{J}=7.3 \mathrm{~Hz}, 2 \mathrm{H}), 0.95(\mathrm{t}, \mathrm{J}=7.4 \mathrm{~Hz}, 3 \mathrm{H}), 0.91(\mathrm{t}, \mathrm{J}=7.4 \mathrm{~Hz}, 3 \mathrm{H}) ;{ }^{13} \mathrm{C}$ NMR $\left(\mathrm{CDCl}_{3}, 100 \mathrm{MHz}\right) \delta 205.6,170.8,61.1,57.4,42.5,32.2,21.4,16.6,13.4,12.1$. Anal. Calcd for $\mathrm{C}_{10} \mathrm{H}_{19} \mathrm{NO}_{3}$ : C, 59.68; H, 9.52; N, 6.96. Found C, 59.90; H, 9.63; N, 7.14 .

(S)-2-Isopropyl-5-methyl-3-oxo-hexanoic acid methoxy-methyl-amide (4b). Acid chloride $=i$ so-valeryl chloride, solvent volume $=10 \mathrm{~mL}$, Time $\mathrm{A}=1 \mathrm{~d}$, Time $\mathrm{B}=1 \mathrm{~d}$, yield $=0.3728 \mathrm{~g}, 65 \%:[\alpha]_{\mathrm{D}}^{23}=-11.0^{\circ}\left(c 1.00, \mathrm{CHCl}_{3}\right)$; IR (neat film) 2959, 1717.2, $1658.9 \mathrm{~cm}^{-1}$; ${ }^{1} \mathrm{H} \mathrm{NMR}\left(\mathrm{CDCl}_{3}, 400 \mathrm{MHz}\right) \delta 3.70(\mathrm{~s}, 3 \mathrm{H}), 3.55(\mathrm{~d}, \mathrm{~J}=9.9 \mathrm{~Hz}, 1 \mathrm{H}), 3.21$ (s, $3 \mathrm{H}), 2.49-2.43(\mathrm{~m}, 2 \mathrm{H}), 2.35(\mathrm{dd}, \mathrm{J}=6.58,17.7 \mathrm{~Hz}), 2.15$ (app septet, $\mathrm{J}=6.62 \mathrm{~Hz}, 1 \mathrm{H})$, 1.01-0.88 (m, 12H); ${ }^{13} \mathrm{C} \mathrm{NMR}\left(\mathrm{CDCl}_{3}, 100 \mathrm{MHz}\right) \delta 205.1,170.0,63.8,61.2,49.6,32.2$, 28.5, 23.5, 22.3, 22.2, 20.8, 20.3. Anal. Calcd for $\mathrm{C}_{12} \mathrm{H}_{23} \mathrm{NO}_{3}$ : C, 62.85; H, 10.11; N, 6.11. Found C, 62.86; H, 10.03; N, 6.38.

Optical Purity Assay for 4b. $\beta$-Ketoamide $4 \mathrm{~b}$ was reduced to the corresponding anti- $\beta$ hydroxyamide with potassium triethylborohydride using the conditions given for $2 .{ }^{2}$ The alcohol was then acylated by treatment with benzoyl chloride, pyridine, and DMAP for 4 $\mathrm{d}$ in $\mathrm{CH}_{2} \mathrm{Cl}_{2}$. HPLC analysis (Daicel Chiralpak OD-H, 99.9:0.01 hexanes:i-propanol, 0.2 $\mathrm{mL} / \mathrm{min}, 254 \mathrm{~nm})$ of the purified compound showed a 98:2 mixture of enantiomers $\left(\mathrm{R}_{\mathrm{t}(S)}\right.$ $=27.21$ min; $\mathrm{R}_{\mathrm{t}(R)}=28.79$.

(S)-2-tert-butyl-5,5-dimethyl-3-oxo-hexanoic acid methoxy-methyl-amide (4c). Acid chloride $=t$-butylacetyl chloride, solvent volume $=10 \mathrm{~mL}$, Time $\mathrm{A}=4 \mathrm{~d}$, Time $\mathrm{B}=2 \mathrm{~d}$, yield $=0.3735 \mathrm{~g}, 58 \%$. HPLC analysis (Daicel Chiralpak OD-H, 99.99:0.01 hexanes: $i$ propanol, $0.5 \mathrm{~mL} / \mathrm{min}, 254 \mathrm{~nm})$ of the purified compound showed a 96:4 mixture of enantiomers $\left(\mathrm{R}_{\mathrm{t}(S)}=17.45 \mathrm{~min} ; \mathrm{R}_{\mathrm{t}(R)}=18.49\right):[\alpha]_{\mathrm{D}}{ }^{23}=+11.4\left(c 1.00, \mathrm{CHCl}_{3}\right) ; \mathrm{IR}$ (neat film) 2954, 1707.4, $1671.6 \mathrm{~cm}^{-1} ;{ }^{1} \mathrm{H} \mathrm{NMR}\left(\mathrm{CDCl}_{3}, 400 \mathrm{MHz}\right) \delta 3.71(\mathrm{~s}, 3 \mathrm{H}), 3.66(\mathrm{~s}, 1 \mathrm{H})$, $3.20(\mathrm{~s}, 3 \mathrm{H}), 2.43(\mathrm{~d}, \mathrm{~J}=17.1 \mathrm{~Hz}, 1 \mathrm{H}), 2.35$ (d, J = $17.0 \mathrm{~Hz}, 1 \mathrm{H}), 1.08$ (s, 9H), 0.98 (s, 9H); ${ }^{13} \mathrm{C} \mathrm{NMR}\left(\mathrm{CDCl}_{3}, 100 \mathrm{MHz}\right) \delta 204.4,170.5,64.0,61.0,54.5,34.7,32.2,30.7,29.3$, 28.3. Anal. Calcd for $\mathrm{C}_{14} \mathrm{H}_{27} \mathrm{NO}_{3}$ : C, 65.33; H, 10.57; N, 5.44. Found C, 65.52; H, $10.72 ; \mathrm{N}, 5.36$.

(S)-3-Oxo-5-triisopropylsilanyloxy-2-triisopropylsilanyloxymethyl-pentanoic acid methoxy-methyl-amide (4d). Acid chloride = 3-(triisopropylsiloxy)propionyl chloride, solvent volume $=50 \mathrm{~mL}$, Time $\mathrm{A}=3 \mathrm{~h}$, Time $\mathrm{B}=2 \mathrm{~h}$, yield $=0.1140 \mathrm{~g}, 88 \%$. HPLC analysis (Daicel Chiralpak OD-H, 99:1 hexanes:i-propanol, $0.5 \mathrm{~mL} / \mathrm{min}, 254 \mathrm{~nm}$ ) of the purified compound showed a 95.5:4.5 mixture of enantiomers $\left(\mathrm{R}_{\mathrm{t}(S)}=9.72 \mathrm{~min} ; \mathrm{R}_{\mathrm{t}(R)}=\right.$ 
10.43): $[\alpha]_{\mathrm{D}}{ }^{23}=+3.2\left(c 1.00, \mathrm{CHCl}_{3}\right) ; \mathrm{IR}$ (neat) 2942.3, 2890.7, 2866.1, 1720.8, 1666.7 $\mathrm{cm}^{-1} ;{ }^{1} \mathrm{H}$ NMR $\left(\mathrm{CDCl}_{3}, 400 \mathrm{MHz}\right) \delta$ 4.19-4.11 (m, 3H), $3.97(\mathrm{t}, \mathrm{J}=6.9 \mathrm{~Hz}, 2 \mathrm{H}), 3.72(\mathrm{~s}$, $3 \mathrm{H}), 3.21(\mathrm{~s}, 3 \mathrm{H}), 2.92(\mathrm{dt}, \mathrm{J}=7.0,17.2 \mathrm{~Hz}, 1 \mathrm{H}), 2.78(\mathrm{dt}, \mathrm{J}=6.6,17.2 \mathrm{~Hz}, 2 \mathrm{H}), 1.20-$ $0.99(\mathrm{~m}, 42 \mathrm{H}) ;{ }^{13} \mathrm{C} \mathrm{NMR}\left(\mathrm{CDCl}_{3}, 100 \mathrm{MHz}\right) \delta 203.9,169.0,62.3,61.3,58.52,58.46$, 45.8, 32.2, 17.9, 17.86, 11.84, 11.78. Anal. Calcd for $\mathrm{C}_{14} \mathrm{H}_{27} \mathrm{NO}_{3}: \mathrm{C}, 60.30 ; \mathrm{H}, 10.70 ; \mathrm{N}$, 2.70. Found C, 60.19; H, 10.83; N, 2.62 .

(S)-3-(Methoxy-methyl-carbamoyl)-4-oxo-heptanedioic dimethyl ester (4e). Acid chloride $=3$-chlorocarbonyl-propionic acid methyl ester, ${ }^{3}$ solvent volume $=50 \mathrm{~mL}$, Time $\mathrm{A}=3 \mathrm{~h}$, Time $\mathrm{B}=2 \mathrm{~h}$, yield $=0.0973 \mathrm{~g}, 64 \%$. HPLC analysis (Daicel Chiralpak OD-H, 82:18 hexanes:i-propanol, $0.5 \mathrm{~mL} / \mathrm{min}, 280 \mathrm{~nm}$ ) of the purified compound showed a $96: 4$ mixture of enantiomers $\left(\mathrm{R}_{\mathrm{t}(S)}=38.79 \mathrm{~min} ; \mathrm{R}_{\mathrm{t}(R)}=42.39\right):[\alpha]_{\mathrm{D}}^{23}=-17.5^{\circ}\left(c 1.00, \mathrm{CHCl}_{3}\right)$; IR (neat) 2954, 1742, $1664 \mathrm{~cm}^{-1}$; ${ }^{1} \mathrm{H} \mathrm{NMR}\left(\mathrm{CDCl}_{3}, 400 \mathrm{MHz}\right) \delta 4.32(\mathrm{dd}, \mathrm{J}=5.4,8.5 \mathrm{~Hz}$, $1 \mathrm{H}), 3.79$ (s, 3H), 3.68 (s, 3H), 3.66 (s, 3H), 3.24 (s, 3H), 2.98 (dd, J = 9, $17.4 \mathrm{~Hz}, 1 \mathrm{H})$, 2.91-2.75 (m, 3H), 2.66-2.52 (m, 2H); ${ }^{13} \mathrm{C}$ NMR $\left(\mathrm{CDCl}_{3}, 100 \mathrm{MHz}\right) \delta 202.2,172.6$, 171.9, 168.9, 61.1, 51.8, 51.6, 51.1, 35.7, 32.3, 31.8, 27.3. Anal. Calcd for $\mathrm{C}_{12} \mathrm{H}_{19} \mathrm{NO}_{7}$ : C, 49.82; H, 6.62; N, 4.84. Found C, 49.71; H, 6.73; N, 4.82 .

\footnotetext{
${ }^{3}$ Ku, T. W.; McCarthy, M.E.; Weichman, B.M.; Gleason, J.G. J. Med. Chem. 1985, 28, 1847-1853.
} 